\title{
Prevalencia de anemia en escolares de la zona amazónica de Ecuador
}

\author{
Edy Quizhpe, ${ }^{1}$ Miguel San Sebastián, ${ }^{1}$ Anna Karin Hurtig ${ }^{1}$ \\ y Ana Llamas ${ }^{1}$
}

RESUMEN Objetivo. Determinar la prevalencia de anemia en niños campesinos de edad escolar en la región amazónica de Ecuador.

Métodos. Se realizó un estudio transversal durante los meses de mayo a octubre de 2000 en dos cantones de la provincia de Orellana, al noreste de Ecuador. Se eligieron 17 escuelas aleatoriamente hasta completar el tamaño muestral deseado, que fue de 626 niños. Se recogieron los datos demográficos y antropométricos (peso y talla); se determinaron los valores de hemoglobina y de protoporfirina eritrocitaria, y se analizaron muestras de heces en busca de infestación por parásitos.

Resultados. La prevalencia general de anemia fue de 16,6\% y de los escolares afectados, $75,5 \%$ tenian anemia por déficit de hierro. La prevalencia de desnutrición crónica moderada fue de $28,8 \%$ y la de desnutrición crónica grave, de 9,3\%. Asimismo, se encontró una prevalencia de desnutrición aguda moderada de 8,4\% y de desnutrición aguda grave de 3,4\%. Las infecciones parasitarias fueron muy frecuentes (82,0\%). Los parásitos más comunes fueron Entamoeba coli $(30,3 \%)$ y Ascaris lumbricoides (25,0\%). No se encontró ninguna relación entre la prevalencia de anemia y anemia por déficit de hierro por un lado, y los indicadores nutricionales o de infección parasitaria por el otro.

Conclusión. La anemia no es un problema grave de salud pública en la población estudiada. No obstante, la elevada prevalencia de niños con desnutrición crónica apunta a la necesidad de mejorar las características de la dieta. La falta de asociación entre la prevalencia de desnutrición y la anemia podría deberse a una baja biodisponibilidad o absorción de hierro, más que a una ingestión insuficiente. Se necesitan estudios que evalúen el tipo de dieta consumida habitualmente por esta población.

Palabras clave Anemia, hierro, escolares, Amazonas, Ecuador.

La anemia es uno de los problemas de salud pública más frecuentes en países en desarrollo (1). Si bien las causas de anemia son multifactoriales, el déficit de hierro se considera el principal

\footnotetext{
1 Instituto de Epidemiología y Salud Comunitaria "Manuel Amunárriz", Quito, Ecuador. Dirección postal: Instituto de Epidemiología y Salud Comunitaria "Manuel Amunárriz", Apdo. 17-10-7410, Nicolás López 349 y Avda. La Prensa, Quito, Ecuador. Teléfono y fax: 5936880 550. Correo electrónico:vaa-ps@ecuanex.net.ec
}

factor responsable de su alta prevalencia $(1,2)$. Numerosos estudios han mostrado que la anemia por déficit de hierro incrementa la morbilidad y la mortalidad en grupos vulnerables, retrasa el crecimiento de los niños y dificulta la función cognoscitiva y el desarrollo escolar $(3,4)$. En los adultos disminuye la capacidad de trabajo y dificulta la labor obstétrica $(5,6)$.

Aunque la anemia por déficit de hierro es más común y grave en los niños menores de 5 años y en las mujeres embarazadas $(7,8)$, este tipo de anemia es también muy frecuente en niños en edad escolar $(9,10)$. En los países en desarrollo, la prevalencia de anemia en escolares se ha estimado en $46 \%$, encontrándose las tasas más altas en África (52\%) y en el sudeste asiático (63\%) (11). En América Latina, el número estimado de niños anémicos en la década de los ochenta del siglo pasado fue de 13,7 millones, lo que equivalía a una prevalencia de $26 \%$ (12). Un informe de la Organización Pana- 
mericana de la Salud (OPS) basado en estudios locales o estatales señaló a Perú como el país con la mayor prevalencia de anemia en toda América Latina y el Caribe (57\%), seguido de Brasil, donde $35 \%$ de los niños de 1 a 4 años estaban anémicos (13).

En América Latina existen, sin embargo, pocos estudios que evalúen la prevalencia de anemia en la población escolar. En un estudio realizado en el altiplano boliviano, se encontró una prevalencia de anemia por déficit de hierro que variaba entre $22 \%$ y $70 \%$ en una población de 0,5 a 9 años de edad (14). En Brasil se encontró una prevalencia de anemia de $26,7 \%$ en niños entre los 7 y 15 años de edad (15). Sin embargo, en un grupo de niños indígenas yaqui de México de 6 a 10 años de edad, tan solo $1,3 \%$ de ellos presentaron anemia (16).

En Ecuador son escasos los estudios sobre la situación de la anemia en los niños. Según los resultados de un estudio de 1996 basado en una muestra representativa de escuelas en zonas de pobreza extrema, $37 \%$ de los escolares tenían anemia, siendo mayor la prevalencia en el primer grado escolar $(45 \%)$ que en el sexto (22\%) (17). En otro estudio sobre la relación entre el estado nutricional y la leishmaniasis cutánea que se realizó en niños entre los 0,5 y los 14 años de edad en un área subtropical del noroeste del país, $12 \%$ de la muestra estaba afectada por anemia por déficit de hierro (18). Vinuesa et al. (19) encontraron una prevalencia de anemia de $32,2 \%$ en niños entre los 6 y 132 meses en la región nororiental del Ecuador.

Cada vez hay un mayor reconocimiento de que los niños en edad escolar, especialmente en los países en desarrollo, sufren de problemas de salud que pueden limitar su capacidad para beneficiarse de la educación $(20,21)$. Por otro lado, el ambiente escolar ofrece un contexto adecuado para efectuar intervenciones de salud pública de diferentes tipos, tales como las que se basan en la educación en materia de salud, en la suplementación de la alimentación con hierro o en la prevención de las infecciones parasitarias (22-24).
El objetivo de este estudio fue determinar la prevalencia de anemia y de deficiencia de hierro en niños campesinos de edad escolar en la región amazónica del Ecuador.

\section{MÉTODOS}

\section{Área de estudio}

La provincia de Orellana, ubicada en el noreste de la región amazónica de Ecuador, consta de cuatro cantones: Francisco de Orellana, Sachas, Loreto y Aguarico. La provincia abarca un área de aproximadamente $20000 \mathrm{~km}^{2}$ y está situada a $300 \mathrm{~m}$ sobre el nivel del mar. El clima de la región es característico de la selva tropical húmeda (precipitación media de $294 \mathrm{~mm}$; temperatura media de $27,9^{\circ} \mathrm{C}$ ). Una humedad de $80 \%$ se mantiene constante en el transcurso del año (25).

La población es de aproximadamente 70000 personas, de las cuales unas 15000 son indígenas y el resto campesinos que en los años 70 llegaron de las regiones costeras o de la Sierra. Aproximadamente un tercio de estos pobladores actualmente viven en ciudades pequeñas donde el comercio y las actividades relacionadas con la industria petrolera son las principales fuentes de ingresos. El resto de la población vive diseminada en diversas comunidades campesinas (con 100 a 400 habitantes) en un área rural tropical, donde una agricultura de subsistencia es la base de la economía. La mayor parte de las comunidades carecen de electricidad y de agua canalizada y tienen una gran dificultad de acceso a los servicios de salud (26). La provincia de Orellana se considera un área donde la transmisión de la malaria es baja y no muestra variaciones estacionales, siendo Plasmodium vivax el parásito prevalente (27).

\section{Diseño del estudio y metodología}

El estudio se realizó durante los meses de mayo a octubre de 2000 en las escuelas fiscales mixtas (educación primaria) de las comunidades campe- sinas localizadas en los cantones de Francisco de Orellana y Sachas, en la provincia de Orellana. El ausentismo escolar es raro y los niños acuden diariamente a las escuelas de sus propias comunidades.

Se elaboró una lista con los nombres y el número de alumnos de todas las escuelas de estos dos cantones que estuvieran registradas en la Dirección Provincial de Educación. El número total de alumnos fue de 6081 . Teniendo en cuenta una prevalencia de anemia en la población escolar de $37 \%$ (17), una precisión deseada de $6 \%$ y un nivel de confianza de $95 \%$, se calculó una muestra de 239 niños. El tamaño de la muestra se duplicó para que se ajustara a la naturaleza del agrupamiento del muestreo. Dando por sentada una participación de $80 \%$, el tamaño de la muestra se incrementó a 598. Diecisiete escuelas se eligieron aleatoriamente hasta completar el tamaño muestral. Todos los niños presentes en cada escuela formaron parte de la muestra.

Un equipo técnico integrado por un médico, una enfermera y un promotor de salud se encargó de recoger los datos y las muestras. Se realizó un estudio piloto en una comunidad elegida al azar. Antes del estudio se obtuvo el consentimiento fundamentado de los padres de familia, los profesores y las autoridades provinciales de salud y educación. Los niños que en el momento del estudio presentaban algún trastorno de salud de manejo primario obtuvieron atención y medicamentos gratuitos.

Datos demográficos. A cada niño o niña se le identificó por su nombre, sexo, edad, el nombre de la escuela y el de la comunidad. La fecha de nacimiento se obtuvo del registro de la escuela o del profesor. Todos los niños registrados estuvieron presentes y fueron examinados, salvo cinco que no participaron en el estudio a solicitud de sus padres.

Análisis de sangre. Se extrajeron $3 \mathrm{~mL}$ de sangre de una vena antecubital con una jeringuilla desechable. La sangre se colocó en un tubo de ensayo con anticoagulante (ácido etilendiaminote- 
tra(a)cético, EDTA) y se transportó refrigerada al Instituto de Biotecnología de la Facultad de Ciencias Médicas de Quito para la determinación de hemoglobina $(\mathrm{Hb})$ y de protoporfirina-cinc (PPC) eritrocitaria. $\mathrm{La} \mathrm{Hb}$ se midió mediante el método de la cianometahemoglobina por espectrofotometría (Eppendorf PCP 6121). Los valores de hemoglobina se estimaron de acuerdo con la corrección de $\mathrm{Hb}$ por altitud y se usaron los puntos de corte para niños establecidos por la Organización Mundial de la Salud y el Grupo Consultivo Internacional sobre Anemia Nutricional (28). Para la altitud de la provincia de Orellana, el punto de corte de la $\mathrm{Hb}$ fue de $11,1 \mathrm{~g} / \mathrm{dL}$. Se definió como anemia grave una concentración de $\mathrm{Hb}$ $<7,0 \mathrm{~g} / \mathrm{dL}$ (1). La PPC se determinó mediante fluorometría (hematofluorómetro AVIV 206) y se utilizó como indicador de la concentración de hierro. Concentraciones elevadas de PPC son indicio de déficit de hierro debido a un suministro inadecuado para la hemosíntesis. Se consideraron normales los valores de PPC $\leq 3 \mu \mathrm{g} / \mathrm{g} \mathrm{Hb}(29)$. Debido a limitaciones económicas, no fue posible medir la ferritina sérica.

Datos antropométricos. Se utilizaron procedimientos normalizados para obtener el peso y la talla (30). El peso se midió a los $500 \mathrm{~g}$ más cercanos con una báscula portátil que se calibró diariamente, y para determinar la estatura se usó un tallímetro de madera adecuadamente calibrado. Los datos se compararon con los del Centro Nacional de Estadísticas de la Salud (31). Los índices de talla para la edad se calcularon en todos los niños que participaron en el estudio, y el índice de peso para la talla se calculó en las niñas hasta los 10 años y en los niños hasta los 11,5 (32). Los niños cuyo índice de talla para la edad estaba situado entre 2 y 3 desviaciones estándar (DE) por debajo de la mediana se clasificaron en la categoría de desnutrición crónica moderada; aquellos cuyos índices se situaban más de 3 DE por debajo de la mediana se clasificaron en la categoría de desnutrición crónica grave. Aquellos cuyos índices de peso para talla estaban entre 2 y 3 DE por debajo de la mediana se clasificaron en la categoría de desnutrición aguda moderada, y los que estuvieron más de $3 \mathrm{DE}$ por debajo de la mediana, en la de desnutrición aguda grave.

Exámenes de heces. Se enseñó a los niños la forma de recoger las heces y se les entregó un pequeño recipiente de plástico con la instrucción de traer una muestra de heces. Se recogieron las muestras y el mismo día las examinó por observación directa un técnico de un laboratorio de Francisco de Orellana reconocido por el Ministerio de Salud Pública. Debido a restricciones logísticas, no fue posible investigar la intensidad de la infección por helmintos.

Examen clínico. A cada niño también se le realizó un examen clínico básico, prestando especial atención a la palidez conjuntival y palmar como signos de anemia (33). Los casos con sospecha de malaria se diagnosticaron clínicamente.

\section{Análisis de los datos}

Todos los datos se analizaron con el programa estadístico EPI-INFO 6.04 (Centers for Disease Control and Prevention, Atlanta, Georgia, Estados Unidos de América). Inicialmente se describieron y se resumieron los datos para documentar su distribución en la población estudiada. Las diferencias entre las variables categóricas se evaluaron mediante las pruebas de ji al cuadrado $\left(\chi^{2}\right)$ y de $\chi^{2}$ para tendencias. Se consideró estadísticamente significativo un valor $P<0,05$.

Para analizar la asociación entre las variables categóricas y la presencia de anemia se utilizó la razón de riesgos o el riesgo relativo (RR) con un intervalo de confianza de $95 \%$.

\section{RESULTADOS}

\section{Características de los participantes}

Se examinaron 626 niños con edades comprendidas entre los 5 y 14 años (media $=8,98$ años; $\mathrm{DE}=2,25$ años). De ellos, 328 (52,4\%) eran de sexo masculino. El grupo étnico más numeroso fue el mestizo $(96,2 \%)$.

\section{Prevalencia de anemia y de deficiencia de hierro}

De los 626 niños examinados, a 592 $(94,6 \%)$ se les realizaron análisis de sangre. La prevalencia total de anemia fue de $16,6 \%$, y en $75,5 \%$ de los casos se encontró anemia por déficit de hierro (cuadro 1). Ciento cincuenta y cinco niños $(26,2 \%)$ tuvieron una PPC $>3 \mu \mathrm{g} / \mathrm{g} \mathrm{Hb}$, pero con un valor de $\mathrm{Hb}$

CUADRO 1. Valores medios de hemoglobina y sus desviaciones estándar (DE) y prevalencia de anemia y de anemia por déficit de hierro, según sexo y edad en una población escolar campesina de la zona amazónica de Ecuador, 2000

\begin{tabular}{|c|c|c|c|c|c|c|}
\hline \multirow[b]{2}{*}{ Población } & \multicolumn{2}{|c|}{$\mathrm{Hb}(\mathrm{g} / \mathrm{dL})$} & \multicolumn{2}{|c|}{$\mathrm{PPC}^{\mathrm{a}}(\mu \mathrm{g} / \mathrm{g} \mathrm{Hb})$} & \multirow{2}{*}{$\begin{array}{c}\text { Anemiab } \\
(\%)\end{array}$} & \multirow{2}{*}{$\begin{array}{c}\text { Anemia por } \\
\text { déficit de hierroc } \\
(\%)\end{array}$} \\
\hline & Media & $(\mathrm{DE})$ & Media & (DE) & & \\
\hline \multicolumn{7}{|l|}{ Sexo } \\
\hline Varones $(n=314)$ & 11,97 & $(1,085)$ & 2,83 & $(1,155)$ & 17,2 & 74,1 \\
\hline Mujeres $(n=278)$ & 12,0 & $(1,019)$ & 2,90 & $(0,968)$ & 15,8 & 77,3 \\
\hline \multicolumn{7}{|l|}{ Edad (años) } \\
\hline $5-7(n=87)$ & 11,70 & $(0,991)$ & 2,97 & $(1,248)$ & 24,1 & 76,2 \\
\hline $7-8,9 \quad(n=165)$ & 11,78 & $(1,061)$ & 2,96 & $(1,119)$ & 23,0 & 84,2 \\
\hline $9-10,9 \quad(n=165)$ & 12,06 & $(0,986)$ & 2,82 & $(0,904)$ & 10,3 & 70,6 \\
\hline $11-12,9(n=140)$ & 12,16 & $(1,053)$ & 2,85 & $(1,058)$ & 15,0 & 61,9 \\
\hline $13-14(n=35)$ & 12,65 & $(1,059)$ & 2,42 & $(1,073)$ & 2,9 & 100 \\
\hline Total $(n=592)$ & 11,99 & $(1,11)$ & 2,87 & $(1,071)$ & 16,6 & 75,5 \\
\hline
\end{tabular}


normal. No se encontró ningún niño con anemia grave.

El porcentaje de niños con anemia fue mayor entre los varones que entre las mujeres, aunque la anemia por déficit de hierro fue ligeramente superior en estas. No hubo diferencias significativas por sexo en ninguno de los indicadores de anemia y de déficit de hierro. Las concentraciones de hemoglobina aumentaron con la edad, encontrándose los valores más bajos entre los 5 y los 9 años. Asimismo, los valores de PPC indicaron que las concentraciones de hierro más bajas se encontraron entre los 5 y los 9 años y aumentaron con la edad (cuadro 1). Esta tendencia de la prevalencia de anemia a reducirse conforme aumenta la edad fue estadísticamente significativa $(P<0,05)$.

En el examen clínico se encontró que $35,1 \%$ de los niños tenían palidez palmar y $17,5 \%$, palidez conjuntival. Se encontró una relación directa significativa entre la anemia y la palidez conjuntival $(P<0,05)$. La sensibilidad del examen de las conjuntivas fue de $27,6 \%$ y la especificidad, de $83,4 \%$. El valor predictivo positivo de la palidez conjuntival fue de $24,8 \%$. Solamente a un niño se le hizo un diagnóstico clínico de malaria.

Estado nutricional. Del total de niños investigados, $273(43,6 \%)$ mostraron indicios de algún tipo de malnutrición. Se encontró una prevalencia de desnutrición crónica (talla para la edad) moderada de $28,8 \%$ (180 casos) y de desnutrición crónica grave, de 9,3\% (58 casos). La prevalencia de desnutrición aguda moderada, expresada en términos del peso para la talla, fue de $8,4 \%$ (37 casos) y la de desnutrición aguda grave, de 3,4\% (15 casos). No se encontraron diferencias significativas por sexo (cuadro 2). Diecisiete niños $(6,2 \%)$ tuvieron indicios de desnutrición crónica y aguda simultáneamente.

\section{Prevalencia de parasitismo}

En total se recogieron $589(94,1 \%)$ muestras de heces y se obtuvieron los resultados de 577 (92,2\%), habiendo

CUADRO 2. Prevalencia de desnutrición moderada y grave según el sexo en una población escolar campesina de la zona amazónica de Ecuador, 2000

\begin{tabular}{|c|c|c|c|c|c|c|}
\hline & \multicolumn{2}{|c|}{ Hombres } & \multicolumn{2}{|c|}{ Mujeres } & \multicolumn{2}{|c|}{ Total } \\
\hline & No. & (\%) & No. & (\%) & No. & $(\%)$ \\
\hline \multicolumn{7}{|c|}{ Talla para la edad $(n=626)$} \\
\hline$<-2 \mathrm{DE}^{\mathrm{a}}$ & 99 & $(30,2)$ & 81 & $(27,2)$ & 180 & $(28,8)$ \\
\hline$<-3 \mathrm{DE}^{\mathrm{b}}$ & 33 & $(10,1)$ & 25 & $(8,4)$ & 58 & $(9,3)$ \\
\hline \multicolumn{7}{|c|}{ Peso para la talla $(n=442)$} \\
\hline$<-2 \mathrm{DE}$ & 20 & $(7,4)$ & 17 & $(10,0)$ & 37 & $(8,4)$ \\
\hline$<-3 \mathrm{DE}$ & 8 & $(2,9)$ & 7 & $(4,1)$ & 15 & $(3,4)$ \\
\hline
\end{tabular}

Nota: $\mathrm{DE}$ = desviación estándar.

a Desnutrición moderada.

b Desnutrición grave.

CUADRO 3. Frecuencia de parasitismo intestinal y tipos de parásitos encontrados en las heces de una población escolar campesina de la zona amazónica de Ecuador, 2000

\begin{tabular}{|c|c|c|c|c|c|c|}
\hline \multirow{2}{*}{$\begin{array}{c}\text { Resultados } \\
\text { de examen de heces }\end{array}$} & \multicolumn{2}{|c|}{ Hombres } & \multicolumn{2}{|c|}{ Mujeres } & \multicolumn{2}{|c|}{ Total } \\
\hline & No. & $\%$ & No. & $\%$ & No. & $\%$ \\
\hline Negativos & 46 & 15,1 & 58 & 21,2 & 104 & 18,0 \\
\hline Positivos & 258 & 84,9 & 215 & 78,8 & 473 & 82,0 \\
\hline \multicolumn{7}{|l|}{ Helmintos } \\
\hline Ascaris lumbricoides & 76 & 25,0 & 68 & 24,9 & 144 & 25,0 \\
\hline Tricocéfalos & 41 & 13,5 & 28 & 10,3 & 69 & 12,0 \\
\hline Anquilostomas & 9 & 3,0 & 13 & 4,8 & 22 & 3,8 \\
\hline Oxiuros & 4 & 1,3 & 1 & 0,37 & 5 & 0,9 \\
\hline Strongiloides & 4 & 1,3 & 1 & 0,37 & 5 & 0,9 \\
\hline \multicolumn{7}{|l|}{ Protozoos } \\
\hline Entamoeba coli & 90 & 29,6 & 85 & 31,1 & 175 & 30,3 \\
\hline Entamoeba hystolitica & 62 & 20,4 & 56 & 20,5 & 118 & 20,5 \\
\hline Giardia lamblia & 42 & 13,8 & 22 & 8,1 & 64 & 11,1 \\
\hline Endolimax nana & 21 & 6,9 & 22 & 8,1 & 43 & 7,5 \\
\hline Otros & 26 & 8,6 & 15 & 5,5 & 41 & 7,1 \\
\hline
\end{tabular}

sido descartadas las demás por ser inadecuadas. Se detectaron infecciones parasitarias en 473 niños (82,0\%). Los parásitos más comunes fueron Entamoeba coli (30,3\%), Ascaris lumbricoides $(25,0 \%)$ y Entamoeba hystolitica $(20,5 \%)$ (cuadro 3). El riesgo de tener parásitos fue mayor en los varones $(P=0,05)$ que en las niñas, pero solo se encontró una relación significativa entre el sexo y la presencia de Giardia lamblia $(P<0,02)$.

\section{Variables relacionadas con la anemia}

No se encontró ninguna relación entre la prevalencia de anemia o de anemia por déficit de hierro y ninguno de los indicadores nutricionales (cuadro 4). Aunque el riesgo de padecer anemia fue mayor en los niños con desnutrición aguda, no fue significativa la diferencia cuando estos niños se compararon con los que tenían desnutrición crónica. Tampoco se halló ninguna relación entre los indicadores de anemia (palidez palmar y conjuntival) y el estado nutricional cuando se restringieron los análisis a los grupos de niños de 10 años o menos y de más de 10 años. No se encontró ninguna relación entre las distintas infecciones por parásitos (salvo en el caso de los anquilostomas) y la concentración plasmática de hierro y de PPC en el grupo 
CUADRO 4. Factores de riesgo asociados con la anemia y con la anemia por déficit de hierro en una población escolar de la zona amazónica de Ecuador, 2000

\begin{tabular}{lccc}
\hline Factor de riesgo & $\begin{array}{c}\text { Prevalencia del } \\
\text { factor de riesgo }\end{array}$ & Riesgo relativo & IC 95\%a \\
\hline Anemia & & & \\
$\quad$ Ascaris & 17,8 & 0,62 & $(0,37-1,02)$ \\
Trichiuris & 13,3 & 1,12 & $(0,65-1,95)$ \\
Anquilostoma & 4,4 & 1,11 & $(0,45-2,76)$ \\
Entamoeba hystolitica & 20,0 & 0,98 & $(0,61-1,58)$ \\
Giardia lamblia & 8,9 & 0,79 & $(0,40-1,56)$ \\
Desnutrición crónica & 30,6 & 0,69 & $(0,46-1,03)$ \\
Desnutrición aguda & 15,6 & 1,55 & $(0,91-2,63)$ \\
Anemia por déficit de hierro & & & \\
Ascaris & 17,4 & 0,60 & $(0,33-1,09)$ \\
Trichiuris & 13,0 & 1,10 & $(0,57-2,10)$ \\
Anquilostoma & 5,8 & 1,47 & $(0,59-3,67)$ \\
Entamoeba hystolitica & 23,2 & 1,19 & $(0,71-2,0)$ \\
Giardia lamblia & 7,2 & 0,64 & $(0,27-1,52)$ \\
Desnutrición crónica & 28,4 & 0,62 & $(0,38-1,0)$ \\
Desnutrición aguda & 16,9 & 1,71 & $(0,94-3,13)$ \\
\hline
\end{tabular}

a Intervalo de confianza de $95 \%$.

de niños menores de 10 años $(P<0,05)$

(datos no mostrados).

\section{DISCUSIÓN}

El presente estudio muestra que la prevalencia de anemia en los niños campesinos en edad escolar de la zona amazónica de Ecuador es de 16,6\%. Esta baja prevalencia es similar a la observada en poblaciones rurales de otras zonas del país (18). Sin embargo, la alta proporción de niños con depósitos bajos de hierro y $\mathrm{Hb}$ normal $(26,2 \%)$ indica que un importante número de participantes está en riesgo de padecer anemia.

La tendencia a que disminuya la prevalencia de anemia y a que aumenten las concentraciones de hierro a medida que aumenta la edad se ha encontrado también en otros estudios $(34,35)$. Una probable explicación es que los niños de menor edad podrían haber tenido recientemente, en la niñez, una mayor demanda de hierro como consecuencia del crecimiento, lo que hace que sus depósitos se reduzcan (10). Al igual que en otros estudios en este grupo de edad, no se encontraron diferencias importantes entre los distintos indicadores de la cantidad de hierro corporal en función del sexo (34, $36,37)$.

Se encontró una relación significativa entre la presencia de anemia y la palidez conjuntival $(P<0,05)$, mientras que la sensibilidad y el valor predictivo positivo del examen de las conjuntivas fueron bajos. Esto significa que la palidez conjuntival no es un indicador fiable de la presencia de anemia leve, aunque sí lo es de la de anemia moderada y grave (38), como han señalado otros estudios. Otros estudios cuyos resultados han sido similares a los nuestros recomiendan valorar la palidez conjuntival para el tamizaje de casos probables, pero no para el diagnóstico (39).

El principal tipo de anemia en este estudio fue por déficit de hierro (75,5\%). Tres motivos podrían explicar la alta prevalencia de anemia por déficit de hierro en nuestra población de estudio: una ingesta insuficiente de hierro en la dieta; una escasa biodisponibilidad o absorción del hierro ingerido, o una pérdida de sangre debido a la presencia de parásitos intestinales (40).

En numerosos estudios se ha observado el importante papel que desempeña la infección por parásitos en este tipo de anemia $(41,42)$. Según estudios realizados en Zanzíbar (África), 25\% de los casos de anemia y 35\% de los casos de anemia por déficit de hierro en niños escolares se atribuían a la infección por anquilostomas (10). Aunque en nuestro estudio todos los niños infectados por anquilostomas tenían anemia por déficit de hierro, la baja prevalencia de este tipo de parasitismo (4\%) descarta la importancia de este factor como causa de anemia en nuestra población. La asociación negativa encontrada entre la anemia y Ascaris lumbricoides también se ha descrito en otros estudios $(10,43)$, y varios autores han observado que la infección por tricocéfalos no causa pérdida de sangre, a pesar de que se ha documentado una asociación entre la presencia de estos parásitos y la concentración hemática de hierro $(44,45)$.

Los protozoos fueron los parásitos más frecuentes en nuestra área, donde $20,5 \%$ de los niños estaban infectados por E. hystolitica. Sin embargo, no se encontró ninguna relación entre la presencia de este parásito y la anemia o el déficit de hierro. Si bien E. hystolitica podría causar diarrea con sangre, no es frecuente encontrar este cuadro ni en la región ni en el grupo de edad estudiados, y sería difícil atribuirle a este parásito la presencia de anemia en el área de estudio (46).

De los distintos factores asociados a la anemia, la ingestión insuficiente de hierro suele ser uno de los más mencionados en la literatura médica $(46,47)$. Aunque en nuestro estudio no se evaluó la ingestión de hierro en la dieta, la población campesina de la región amazónica mantiene una dieta constante basada en carbohidratos (arroz, yuca, plátano), pero la ingestión de proteínas y de frutas es también habitual. A pesar de la alta prevalencia de desnutrición crónica observada en los niños de esta edad $(38,1 \%)$, los indicadores antropométricos no mostraron asociación con la anemia ni con la anemia por déficit de hierro. Este hecho apunta a que la causa inmediata del déficit de hierro podría no ser una ingestión insuficiente tanto como una baja biodisponibilidad o absorción. Diferentes estudios han mostrado que las dietas en los países en desarrollo carecen de variedad y contienen sustancias que pueden inhibir la 
absorción de hierro, como son los taninos, los fenoles y la fibra, componentes frecuentes de las dietas ricas en carbohidratos, como la de la región estudiada $(46,48)$.

El presente estudio muestra que la anemia no es un problema grave de salud pública en la zona amazónica de Ecuador. Sin embargo, es importante destacar la gran proporción de niños en riesgo de padecer anemia por agotamiento de sus depósitos de hierro. El elevado porcentaje de niños con desnutrición crónica apunta a un déficit de la ingestión de macro y micronutrientes $(46,49)$ y, por tanto, a la nece- sidad de mejorar las características de la dieta.

Para determinar si la anemia por déficit de hierro hallada en esta población se debe o no a un problema de biodisponibilidad o absorción se necesitan estudios más profundos que definan los factores de riesgo de la anemia por déficit de hierro y el tipo de dieta habitualmente consumida por la población estudiada. Por otro lado, el gobierno ecuatoriano ha iniciado recientemente en las escuelas de la región amazónica un programa de fortificación de alimentos con hierro. El programa debe vigilarse con miras a evaluar su impacto.
Agradecimientos. Este estudio no podría haberse realizado sin la colaboración de los maestros, los padres de familia y los niños de las comunidades estudiadas. Agradecemos a Juan Carlos Freire, así como al equipo técnico y a los promotores de salud de la Fundación Salud Amazónica, su apoyo logístico; al Instituto de Biomedicina de la Universidad Central el procesamiento de las muestras; y al Dr. Juan Antonio Córdoba sus valiosos comentarios a una versión previa de este manuscrito. Este estudio fue financiado con el apoyo de Medicus Mundi Guipúzcoa y Capuchinos Navarra.

\section{REFERENCIAS}

1. Demaeyer E. Preventing and controlling iron deficiency anaemia through primary health care. A guide for health administrators and programme managers. Geneva: World Health Organization; 1989.

2. United Nations Children's Fund. The state of the world's children. New York: UNICEF; 1998.

3. Pollitt E, Leibel R, Greenfield DB. Iron deficiency and cognitive test performance in preschool children. Nutr Behav 1983;1:137- 146.

4. Angeles IT, Schultink WJ, Matulessi P, Gross $\mathrm{R}$, Sastroamidjojo S. Decreased rate of stunting among anemic Indonesian preschool children through iron supplementation. Am J Clin Nutr 1993;58:339-342.

5. Garn SM, Keating MT, Falkner F. Hematological status and pregnancy outcomes. Am J Clin Nutr 1981;34:115-117.

6. Scrimshaw N. Iron deficiency. Sci Am 1991; 265(4):46-52.

7. Charlton RW, Bothwell TH. Definition, prevalence and prevention of iron deficiency. Clin Haematol 1982;11:309-325.

8. United Nations, ACC/SCN. Controlling iron deficiency. New York: United Nations; 1991. (Nutrition Policy Discussion Paper No. 9).

9. Prual A, Daouda H, Develoux M, Sellin B, Galan P, Hercberg S. Consequences of Schistosoma haematobium infection on the iron status of schoolchildren in Níger. Am J Trop Med Hyg 1992;47:291-297.

10. Stoltzfus RJ, Chwaya HM, Tielsch JM, Schulze KJ, Albonico M, Savioli L. Epidemiology of iron deficiency anemia in Zanzibari schoolchildren: the importance of hookworm. Am J Clin Nutr 1997;65:153-159.

11. Administrative Committee on Coordination, Sub-committee on Nutrition. Fourth Report on the World Nutrition Situation, 2000. United Nations. Geneva: ACC/SCN; 2000.

12. Horwitz A. El costo de la malnutrición. En: Organización Panamericana de la Salud, eds. Vigilancia alimentaria y nutricional en las
Américas. Washington, D.C.: Organización Panamericana de la Salud; 1989. Pp. 19-54. (Publicación Científica, 516).

13. Mora JO, Mora LM. Deficiencias de micronutrientes en América Latina y el Caribe: anemia ferropriva. Washington, D.C.: Organización Panamericana de la Salud; 1997.

14. Berger J. L'anémie chez l'enfant dans les pays en development. Étude de deux modèles au Togo et en Bolivie. [Tesis de doctorado]. Université de Montpellier, France; 1996.

15. Tsuyuoka R, Bailey JW, Nery Guimaraes AM, Gurgel RQ, Cuevas LE. Anemia and intestinal parasitic infections in primary school students in Aracaju, Sergipe, Brazil. Cad Saude Publica 1999;15(2):413-421.

16. Valencia ME, Astiazaran $H$, Esparza J, Gonzalez L, Grijalva MI, Cervera A, et al. Vitamin A deficiency and low prevalence of anemia in Yaqui Indian children in northwest Mexico. J Nutr Sci Vitaminol 1999;45(6):747-757.

17. Pan American Health Organization. Health in the Americas. Washington, D.C.: PAHO; 1998.

18. Weigel MM, Armijos RX, Zurita C, Racines J, Reddy A, Mosquera J. Nutritional status and cutaneous leishmaniasis in rural Ecuadorian children. J Trop Ped 1995;41:22-28.

19. Vinuesa R, Dávila M, Estévez E, Yépez F, Racines $\mathrm{M}$, Vivero $\mathrm{S}$, Séller $\mathrm{P}$, et al. La uncinariasis como factor de riesgo para anemia en niños de dos regiones tropicales del Ecuador. IV Congreso Latinoamericano de Medicina Tropical. Quito; 1993.

20. Pollitt E. Malnutrition and infection in the classroom. Paris: United Nations Educational, Scientific and Cultural Organization; 1990.

21. Sternberg RJ, Grigorenko EL, Nokes C. Effects of children's ill health on cognitive development. En: Young ME, ed. Early child development. Washington, D.C.: World Bank; 1997. Pp. 85-125.

22. Walter T, Hertrampf E, Pizarro F, Olivares M, Llaguno S, Letelier A, et al. Effect of bovinehemoglobin-fortified cookies on iron status of schoolchildren: a nationwide program in Chile. Am J Clin Nutr 1993;57(2):190-194.

23. Cyrino EG, Pereira ML. Reflexões sobre uma proposta de integração salud-escola: o projeto saude e educação de Botucatu, São Paulo. Cad Saude Publica 1999;15(Suppl 2):39-44.

24. Magnussen $P$, Muchiri E, Mungai $P$, Ndzovu M, Ouma J, Tosha S. A school-based approach to the control of schistosomiasis and intestinal helminth infections in children in Matuga, Kenya: impact of a two-year chemotherapy programme on prevalence and intensity infections. Trop Med Int Health 1997; 9:825-831.

25. Fundación José Peralta. Ecuador: su realidad. Quito: Fundación de Investigación y Promoción Social José Peralta; 2001.

26. Tamariz ME, Villaverde X. Diagnóstico de la tenencia de la tierra en las provincias de Napo y Sucumbios. Fepp: Quito; 1997.

27. San Sebastian M, Jativa R, Goicolea I. Epidemiology of malaria in the Amazon basin of Ecuador. Rev Panam Salud Publica 2000;7(1): 24-28.

28. International Nutritional Anemia Consultative Group (INACG). Measurement of iron status. Washington, D.C.: The International Nutritional Anemia Consultative Group; 1985.

29. Arija V, Fernández J, Salas J. Carencia de hierro y anemia ferropénica en la población española. Med Clin 1997;109:425-430.

30. Jelliffe DB. The assessment of the nutritional status of the community. WHO Monograph Series 63. Geneva: World Health Organization; 1966.

31. National Center for Health Statistics. NCHS growth curves for children birth -18 years. Washington, D.C.: US Department of Health, Education and Welfare; 1977.

32. WHO Working Group. Use and interpretation of anthropometric indicators of nutritional status. Bull World Health Organ 1986;64:929941. 
33. Getaneh T, Girma T, Belachew T, Teklemariam $\mathrm{S}$. The utility of pallor detecting anemia in under five years old children. Ethiop Med J 2000;38(2):77-84.

34. Tatala S, Svanberg U, Mduma B. Low dietary iron availability is a major cause of anemia: a nutrition survey in the Lindi District of Tanzania. Am J Clin Nutr 1998; 68:171-178.

35. Murila FV, Macharia WM, Wafula EM. Iron deficiency anaemia in children of a peri-urban health facility. East Afr Med J 1999;76(9):520523.

36. Robertson LJ, Crompton DWT, Sanjur D, Nesheim MC. Haemoglobin concentrations and concomitant infections of hookworm and Trichuris trichiura in Panamanian primary schoolchildren. Trans $\mathrm{R}$ Soc Trop Med Hyg 1992;86:654-656.

37. Ahmed F, Barua S, Mohiduzzaman M, et al. Interactions between growth and nutrient status in school-age children of urban Bangladesh. Am J Clin Nutr 1993; 58:334-338.

38. Zucker JR, Perkins BA, Jafari H, Otieno J, Obonyo C, Campbell CC. Clinical signs for the recognition of children with moderate or severe anaemia in western Kenya. Bull World Health Organ 1997;75(Suppl 1):97-102.
39. Ekunwe EO. Predictive value of conjunctival pallor in the diagnosis of anaemia. West Afr J Med 1997;16(4):246-250.

40. Cook JD, Skikne BS, Baynes Rd. Iron deficiency: the global perspective. Progress in iron research. New York: Plenum Press; 1994. Pp. 219-228.

41. Kinoti SN. Epidemiology of hookworm disease and iron deficiency anaemia in Kenya. East Afr Med J 1982;59:341-344.

42. Stephenson LS, Kinoti SN, Latham MC, Kurz KM, Kyobe J. Single dose metrifonate or praziquantel treatment in Kenyan children. I. Effects on Schistosoma haematobium, hookworm, hemoglobin levels, splenomegaly and hepatomegaly. Am J Trop Med Hyg 1989;41: 436-444.

43. Curtale R, Tilden R, Muhilal, Vaidya Y, Pokhrel RP, Guerra R. Intestinal helminths and risk of anaemia among Nepalese children. Panminerva Med 1993;58:334-338.

44. Greenberg ER, Cline BL. Is trichuriasis associated with iron deficiency anemia? Am J Trop Med Hyg 1979;28:770-772.

45. Stoltzfus RJ, Albonico M, Chwaya HM, et al. Hemoquant determination of hookworm related blood loss and its role in iron deficiency in
African children. Am J Trop Med Hyg 1996; 55:399-404.

46. Adish AA, Esrey SA, Gyorkos TW, Johns T. Risk factors for iron deficiency anaemia en preschool children in northern Ethiopia. Public Health Nutr 1999; 2(3): 243-252.

47. Skikne BS. Current concepts in iron deficiency anaemia. Food Rev Int 1988;4:137-173.

48. Haghshenass M, Mahloudij M, Reinhold JG, Mohammadi N. Iron-deficiency in an Iranian population associated with high intakes of iron. Am J Clin Nutr 1972;25: 1143-1146.

49. Pollitt E. Functional significance of the covariance between protein energy malnutrition and iron deficiency anemia. J Nutr 1995;46: $465-473$.

Manuscrito recibido el 22 de mayo de 2002. Aceptado para publicación, tras revisión, el 17 de septiembre de 2002.

ABSTRACT Objective. To determine the prevalence of anemia in rural school-age children in the Amazon region of Ecuador.

Methods. We carried out a cross-sectional study during May to October 2000 in two cantons of the province of Orellana, in northeastern Ecuador, involving 626 children from 17 schools. Demographic and anthropometric data (weight and height) were collected, values for hemoglobin and for zinc erythrocyte protoporphyrin were determined, and feces samples were analyzed to check for infestation by parasites.

Results. The general prevalence of anemia was $16.6 \%$ among the schoolchildren; of the affected children, $75.5 \%$ of them had iron-deficiency anemia. The prevalence of moderate chronic undernutrition was $28.8 \%$ and that of serious chronic undernutrition was $9.3 \%$. There was also a prevalence of moderate acute undernutrition of $8.4 \%$ and of severe acute undernutrition of $3.4 \%$. Parasitic infections were very frequent $(82.0 \%)$. The most common parasites were Entamoeba coli (30.3\%) and Ascaris lumbricoides $(25.0 \%)$. There were no relationships between the prevalence of either anemia or of iron-deficiency anemia and any of the indicators of nutrition or of parasitic infection.

Conclusions. Anemia is not a serious public health problem in the population studied. Nevertheless, the high prevalence of chronic undernutrition among the children points to the need to improve their diets. The lack of association between the prevalence of undernutrition and anemia could be due to low iron bioavailability or absorption rather than insufficient intake. Studies are needed to evaluate the customary diet of this population. 